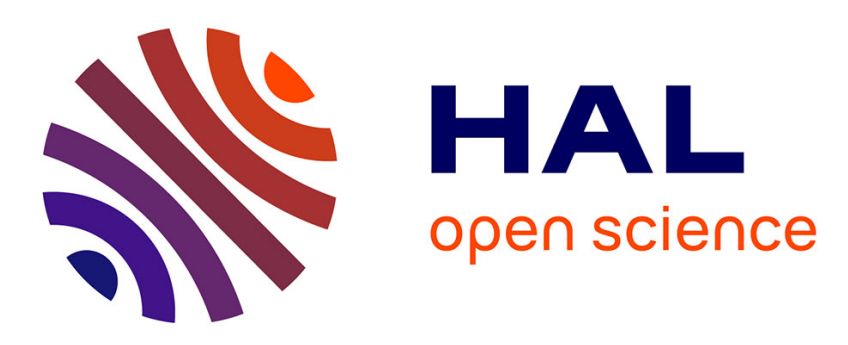

\title{
Noise- and dynamics-sustained patterns in a nonlinear photorefractive system
}

\author{
Nicolas Marsal, Delphine Wolfersberger, Marc Sciamanna, Germano \\ Montemezzani
}

\section{- To cite this version:}

Nicolas Marsal, Delphine Wolfersberger, Marc Sciamanna, Germano Montemezzani. Noise- and dynamics-sustained patterns in a nonlinear photorefractive system. Physical Review A: Atomic, molecular, and optical physics [1990-2015], 2010, 81 (3), pp.031804(R)-1-4. 10.1103/PhysRevA.81.031804. hal-00474394

\section{HAL Id: hal-00474394}

https://hal-centralesupelec.archives-ouvertes.fr/hal-00474394

Submitted on 4 Jun 2021

HAL is a multi-disciplinary open access archive for the deposit and dissemination of scientific research documents, whether they are published or not. The documents may come from teaching and research institutions in France or abroad, or from public or private research centers.
L'archive ouverte pluridisciplinaire HAL, est destinée au dépôt et à la diffusion de documents scientifiques de niveau recherche, publiés ou non, émanant des établissements d'enseignement et de recherche français ou étrangers, des laboratoires publics ou privés. 


\title{
Noise- and dynamics-sustained patterns in a nonlinear photorefractive system
}

\author{
N. Marsal, ${ }^{*}$ D. Wolfersberger, M. Sciamanna, and G. Montemezzani \\ LMOPS Laboratory, Supelec and Université Paul Verlaine de Metz, France
}

(Received 29 September 2009; published 29 March 2010)

\begin{abstract}
We analyze experimentally the dynamics of optical patterns emerging from a photorefractive two-wave mixing geometry where a tilted single-feedback mirror generates an advectionlike effect. Depending on the nonlocal coupling (introduced by the tilting angle) between the two counterpropagating beams, the strength of the nonlocal response of the nonlinear photorefractive bulk medium, and the distance between the mirror and the crystal, we observe the initiation of different pattern geometries, the inversion of pattern transverse phase velocity, and the bifurcation from convective to absolute instabilities.
\end{abstract}

DOI: 10.1103/PhysRevA.81.031804

PACS number(s): 42.65.Sf, 47.54.-r, 05.40.Ca, 05.45.-a

Because of light-matter interaction, laser beams propagating through nonlinear media can undergo peculiar effects, generating a variety of known or new physical mechanisms [1]. Among these many mechanisms, the effect of noise can drive the system out of the homogeneous state, which can become unstable because of modulational instability (MI), leading to the formation of spatial instabilities called patterns [2,3]. For its potential application in optical memory, spontaneous pattern formation has been studied in different nonlinear optical systems [4-10]. In general, such dissipative systems display a large number of unstable pattern modes, but only a few of them are selected such as stripes and hexagons.

The pattern selection intrinsically implies a spontaneous breaking of the translation and rotation symmetry in the system. An additional intentional symmetry breaking by an external influence of the space symmetry is therefore expected to modify the pattern geometry. To that end, a breaking of the reflection symmetry has been proved to be an efficient process [11-15]. Such a breaking arises if the feedback pump beam is tilted in the system, giving rise to an advectionlike effect. Because of this advection, patterns will experience different behaviors, not only on their geometry but also on their dynamics. Indeed, this latter effect can become either convective or absolute [16], depending on various parameters. Basically, in the so-called "convective regime," a perturbation growing on a homogeneous state is simultaneously advected away so that the system returns to the initial homogeneous solution: No pattern can arise in this regime. In contrast, in the absolute regime, a disturbance growing locally can compete with the advection so that the system reaches a pattern state. However, the convective regime, where no pattern is expected, can exhibit patterns if noise is present in the system. Then, macroscopic noise-sustained structures can be formed as a result of the amplification in preferential direction of the perturbations produced by the microscopic noise [17].

Advectionlike effects leading to convective instabilities have been modeled initially by drift terms in the governing equations of different systems [17]. This approach is valid for small shifts of the feedback beam. It has also been demonstrated, in the general case where large spatial shifts (that cannot be approximated by drift terms) are allowed, that the nonlocal spatial coupling opens a larger range of parameters

\footnotetext{
*nicolas.marsal@supelec.fr
}

where noise-sustained patterns should exist. In these cases, one speaks often about "two-point nonlocality" $[18,19]$.

Obviously, both models contribute to the study of convective instabilities and, recently, convective regimes have been reported experimentally in a single-feedback Kerr-type medium [20,21]. Numerical studies in a temporal system with mismatched synchronization of the pump pulses in a fiber ring cavity [22] and theoretical works with lasers with nonlocal feedback [23] have also provided evidence for such convective phenomena.

In spite of the extensive theoretical interest, experimental demonstrations of such convective dynamics remain scarce and focused mostly on Kerr-type nonlinear media. In this article, we investigate experimentally the dynamics of pattern excitation in the presence of an advectionlike effect in a nonlinear photorefractive single-feedback system. When modifying the angle of the feedback mirror and when properly adjusting the intensity of the pump beam $\left(\mathrm{I}_{P}\right)$, we observe how the strength of the advection can (i) influence the geometry of the forming patterns, (ii) seed noise-sustained structures, (iii) inverse the transverse phase velocity of a drifting pattern, and (iv) enable the bifurcation from convective (CI) to absolute (AI) instability modes. It is worth noting that our study concerns a nonlinear thick medium that exhibits two kinds of nonlocalities: the nonlocal photorefractive response and the nonlocal spatial coupling between the two counterpropagating beams introduced by the misalignment in the feedback loop. In our experiment, the latter can be large and can be theoretically modeled by a "nonlocality" (shift) in the equations of the photorefractive system. In this article we concentrate on the experimental observations of the effects resulting from such nonlocality.

Our experimental setup (Fig. 1) is similar to the configuration described in Refs. [20,24]. It contains a standard photorefractive two-wave mixing in a reflection-grating geometry and a tunable single feedback. A $p$-polarized 532-nm laser beam is focused inside a nominally undoped $\mathrm{BaTiO}_{3}$ crystal to a $350-\mu \mathrm{m}$ diameter. The feedback mirror can be precisely moved longitudinally to vary the position of the corresponding virtual mirror created by a $2 f: 2 f$ lens system. This makes it possible to adjust positive (mirror outside the medium) or negative (mirror inside the medium) effective propagation lengths $L$ (Fig. 1) [25].

In this counterpropagating configuration, for a particular intensity threshold $\mathrm{I}_{\mathrm{Th}}$, the pump beam becomes unstable 


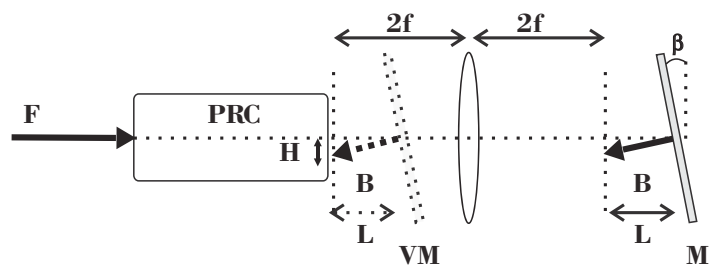

FIG. 1. Schematic of the setup composed of a photorefractive crystal (PRC) in a single-feedback configuration. M, mirror; VM, virtual mirror; L, distance between the virtual mirror and the crystal; $\beta$, mirror tilt angle; $H=L \sin \beta$, transverse shift (in $\mu \mathrm{m}$ ); $\mathrm{F}$ and $\mathrm{B}$ correspond respectively to the forward and backward beams responsible for the pattern formation.

against MI. The pump intensity is correlated to the gain of the (nonlocal) reflection grating arising in the crystal due to photorefractive effect [26]. Above $I_{\mathrm{Th}}$, MI leads to the formation of patterns [5,24], which are identified by monitoring their far and near fields onto a CCD camera. Depending on the distance between the mirror and the crystal ( $L$ in Fig. 1) and above $\mathrm{I}_{\mathrm{Th}}$, a finite set of wave vectors pairs is selected, leading to the formation of different patterns, with hexagonal being the main one. Its far- and near-field intensity distributions are shown in Fig. 2(A). To create an advectionlike effect, the feedback mirror can be precisely tilted in the $x$ or $y$ direction, giving rise to the shift $H$ in Fig. 1 .

Let us first analyze the behavior related to the formation of different patterns geometries in our system. We arbitrarily choose the transverse displacement $H$ along the $y$ axis. For a feedback mirror located outside the medium in the absence of advection $(H=0)$, hexagons are the only displayed patterns [Fig. 2(A)]. With increasing the transverse shift, we successively observe horizontal stripes [Fig. 2(B)], squeezed hexagons [Fig. 2(C)], and eventually a state where no clearly defined pattern exists [Fig. 2(D)], although vestiges of the previous horizontal lines are still present in the near field. This scenario follows the one observed in [25].

The scenario changes when the virtual mirror is located inside. Thereby, starting from the situation in which the transverse shift $H$ is absent [Fig. 3(A)], we increase the value of $H$ and successively discover different patterns similar to those observed earlier in liquid-crystal Kerr-type media [14,21]: horizontal stripes, squeezed hexagon, vertical stripes, rectangular lattice, no pattern [Figs. 3(B)-3(F)]. Similar

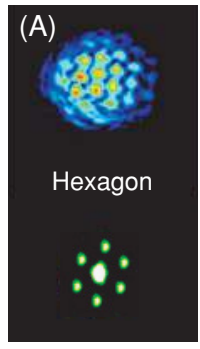

$\mathrm{H}=0$

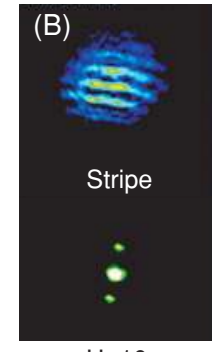

$\mathrm{H}=10$

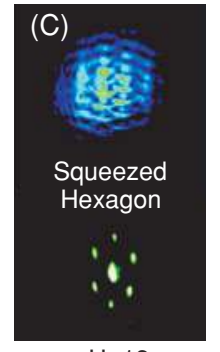

$\mathrm{H}=18$

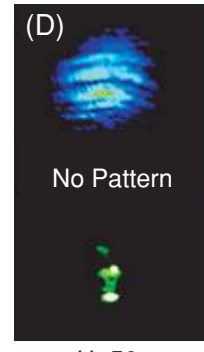

$\mathrm{H}=56$
FIG. 2. (Color online) Different patterns obtained for different transverse shifts $H$ (in $\mu \mathrm{m}$ ) and for a virtual mirror located outside the $\operatorname{crystal}(L \approx 1 \mathrm{~mm})$. The pump beam intensity is fixed to the value $I_{p} \simeq 45 \mathrm{~mW}$. Top, near field; bottom, far field.
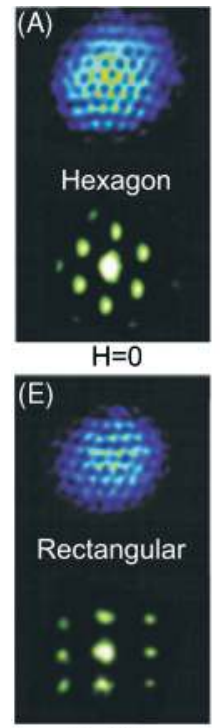

$\mathrm{H}=33$
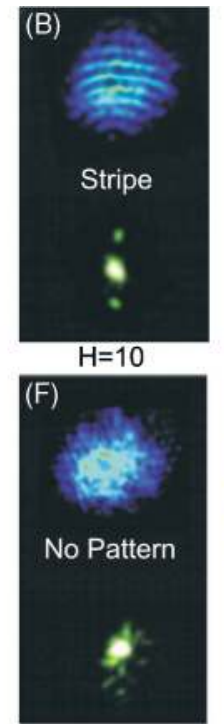

$\mathrm{H}=63$
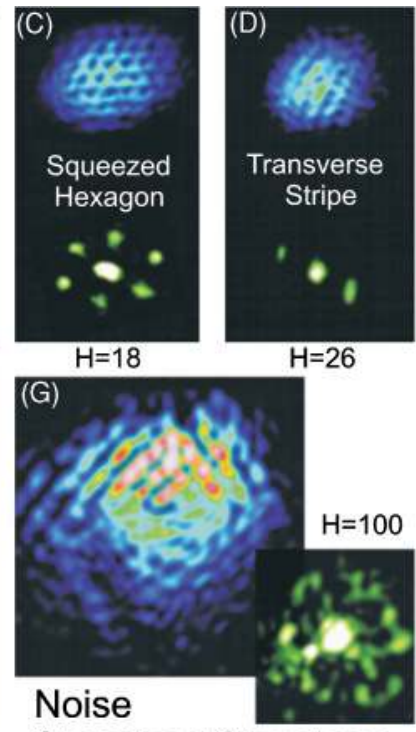

Sustained Structures
FIG. 3. (Color online) Different patterns obtained for different transverse shifts $H$ (in $\mu \mathrm{m}$ ) and for a virtual mirror located inside the crystal $(L \approx-2 \mathrm{~mm}$ ). The pump beam intensity is fixed to the value $I_{p} \simeq 45 \mathrm{~mW}$. Top, near-field; bottom, far-field.

patterns were already observed in such a nonlinear system by modifying the longitudinal position of the mirror in the so-called "multiple-patterns" region inside the medium [25]. However, in our case these different patterns are obtained by changing the mirror angle (reflection breaking) when the virtual mirror has a fixed position inside the multiple-patterns region. Furthermore, a different peculiar structured state is observed in Fig. 3(G). It is obtained for large values of $H$ and does not depend drastically on $I_{p}$. This disordered state does not occupy the whole available space and is located at the edge of the outgoing flow created by the strong misalignment. The corresponding far field shows noisy hexagonal spots located all around a ring of instabilities. This behavior corresponds to typical noise-sustained structures, as predicted in Fig. 6 of Ref. [27], for large values of the parameter $H$.

The profile of each pattern, observed in presence of an advectionlike effect (Figs. 2 and 3), propagates along the transverse shift direction with a transverse group velocity (velocity of the envelope of the pattern) always positive and increasing with $H$. In fact, each pattern exists for a range of parameter $H$, and inside this range the transverse group velocity increases with $H$ until another pattern state is reached. Nevertheless, even if the propagation of the pattern envelope is directed toward the direction of the transverse shift, the phase velocity (velocity of the pattern components) can be positive or negative and even equal to zero. This remarkable nonintuitive property means that the pattern components can drift downstream, drift upstream, or stabilize themselves, depending on $H$ [19,21]. We show an example of this phenomenon in our system (Fig. 4)], for the squeezed hexagon obtained in Fig. 2(C). For the range of parameters, $18<H<30$, where this pattern appears, we find that the envelope and the components of the pattern follow the transverse direction of the shift imposed by the orientation of the mirror (vertical arrow in the leftmost panel of Fig. 4): Both the phase and group velocities are positive 

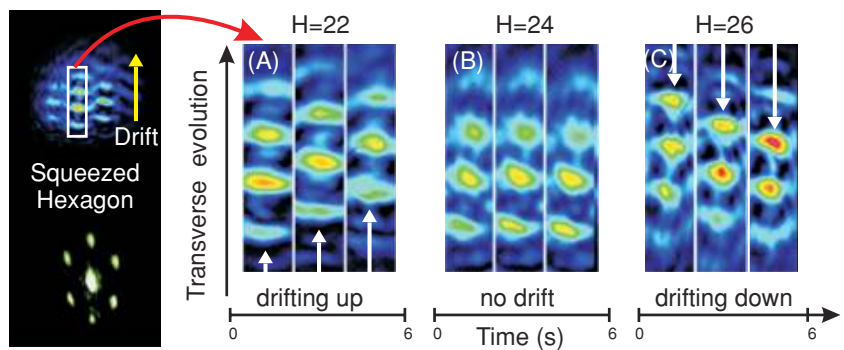

FIG. 4. (Color online) Time evolution of the transverse profile of the squeezed hexagon from Fig. 2(C). From left to right, drifting pattern passing from a positive transverse phase velocity $v \simeq+\pi /$ $3 \mathrm{rad} \mathrm{s}^{-1}$ (A) to null (B) then to a negative value $v \simeq-\pi / 3 \mathrm{rad} \mathrm{s}^{-1}$ (C) for different $H$.

[Fig. 4(A)]. Then, for a particular value of $H[H=24$ in Fig. 4(B)], the pattern stabilizes itself (null phase velocity) before moving in the other direction $(H=26)$, although the shift is always imposed in the opposite direction: The phase velocity is henceforth negative [Fig. 4(C)].

Let us now identify the different dynamics in the patternformation process. As explained, the dynamics can be either convective or absolute; both possess their own unique signatures [17-22,27,28]. Indeed, in the near-field, noise-sustained patterns must not occupy all the system space but are rather located in the area closest to the edge of the outgoing flow, in contrast to dynamics-sustained patterns which invade the whole space. A time-resolved spectral analysis of field dynamics can quantitatively determine the nature of the pattern (dynamics- or noise-sustained). Finally, the analysis of the width and shape of the near- and far-field patterns makes it possible to find the thresholds of the transition from CI to AI.

Experimentally, as seen earlier in this article, two main features must be taken into account: the near- and far-field spatiotemporal intensity evolution of the pattern (Fig. 5) and the relative size occupied by the pattern (Fig. 6). We choose the stripe pattern from Fig. 2(B). For increasing pump power, we observe three qualitatively different behaviors similar to those seen in Kerr-type material [20].

(i) For low input intensities, the output beam shows erratic stripes that appear randomly in time and space with short time duration [Fig. 5(A)]. This is due to the fact that noise excites all possible orientations and the newly generated advected pattern has no relation with the one already formed. Regarding the space occupation of the near field for low-intensity power (Fig. 6, "NP" area), the stripes occupy on average $80 \%$ of the available space. In addition, the two corresponding spots in the far-field [Fig. 5(A), inset] are very broad and move in time. All these observations are associated with a precursor, induced by noise, of the pattern that emerges for high intensities in the absolute regime [28].

(ii) When the input intensity is increased up to $18 \mathrm{~mW}$, stripes, drifting outside the pump beam [Fig. 5(B)], arise continuously. This structure occupies only $60 \%$ of the available space and have a spread width increasing slowly with $I_{p}$ (Fig. 6, "CI" area). The far-field pattern is now stable in its transverse plane, but still present extended spots [inset Fig. 5(B)]. In Fig. 5(B), the pattern arises remarkably at a different spatial position for different times. In this case,

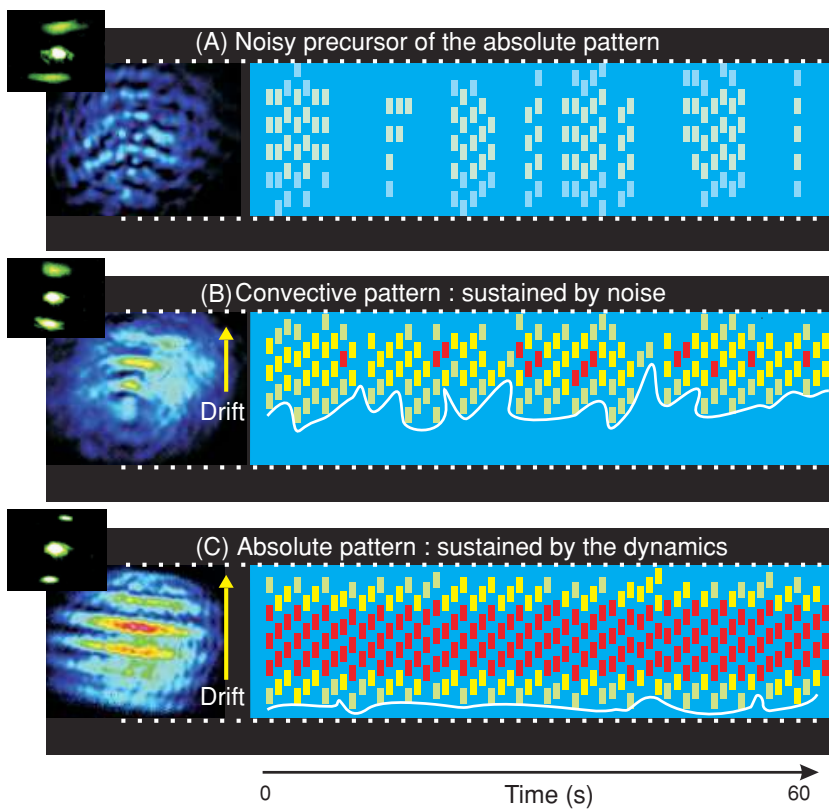

FIG. 5. (Color online) Spatiotemporal evolution of the transverse profile of the stripe pattern obtained in Fig. 2(B) $(H=10 \mu \mathrm{m})$ for different powers of the pump beam: (A) $15 \mathrm{~mW}$, (B) $30 \mathrm{~mW}$, (C) $55 \mathrm{~mW}$. The dotted lines depict the available space for the growing process of the pattern. From blue to red, the colors indicate respectively an evolution from low to high intensities in the near-field pattern. (Inset) Corresponding far field.

the perturbations are amplified, are advected away [drift in Fig. 5(B)], and then reappear recursively. All these behaviors characterize a regime of noise-sustained pattern. Note that Fig. 3(G), obtained for a stronger advection force, shares similar behaviors with this regime, but no particular pattern is obtained during the process. Such convective instabilities would not have been observed if noise had not been present in the system: this regime is known to be convective, sustained by noise.

(iii) For high input intensities, the stripe pattern extends further upstream and invades almost all the available pumping region of the system [Fig. 5(C); Fig. 6, "AI" area]. The

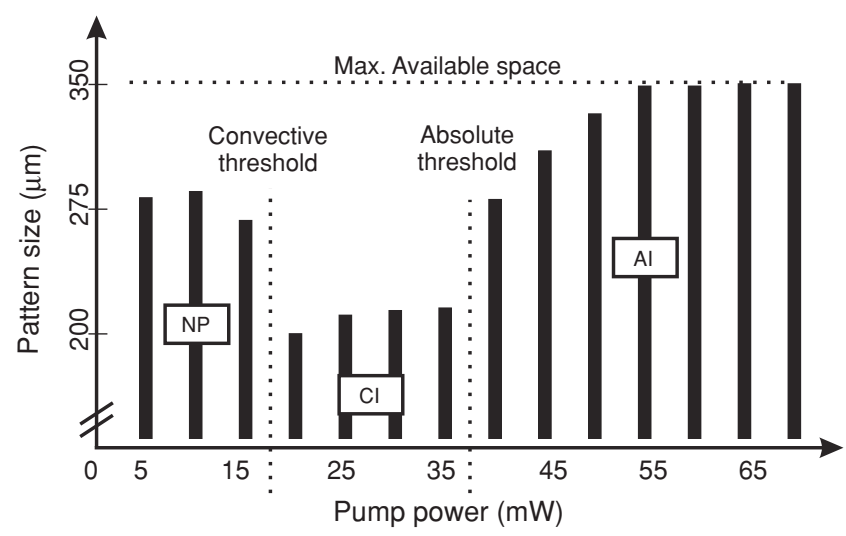

FIG. 6. Evolution of near-field pattern size versus the pump power. NP, noisy precursor regime; CI, convective instability regime; AI, absolute instability regime. 
spatiotemporal trace shows a strong periodicity of the advection effect. The far-field spots [Fig. 5(C), inset] are now well-defined. Finally, by comparison with the convective regime, note that the pattern arises almost at the same position in space [see the white lines following the upstream edges of the stripe pattern in Figs. 5(B) and 5(C)]. These phenomena are linked to an absolute regime displaying dynamics-sustained patterns.

Finally, Fig. 6 shows the evolution of the mean width of the pattern region as a function of the pump intensity. The three regimes discussed earlier in this article (noisy precursor, convective instability, absolute instability) manifest themselves when the pump intensity reaches different thresholds and clearly correspond to different signatures of the pattern dynamics and of the corresponding pattern size [20]. Indeed, in Fig. 6 we identify three different plateaus located between two critical values (vertical dashed lines) of the pump beam intensity: the convective and absolute intensity thresholds, respectively. Before the first division in the graph $\left(\mathrm{CI}\right.$ threshold at $I_{p} \simeq 18 \mathrm{~mW}$ ), the first plateau settles in a structure that occupies approximately $80 \%$ of the available space $(\simeq 350 \mu \mathrm{m})$ and represents the noisy precursor (NP) of the dynamics-sustained pattern [case (i)]. After the second plateau (AI threshold at $I_{p} \simeq 37 \mathrm{~mW}$ ), the pattern starts to invade the whole system and reaches a saturated value (above $I_{p} \simeq 55 \mathrm{~mW}$ ): here we are in the AI regime [case (iii)]. Finally, between $I_{p} \simeq 18 \mathrm{~mW}$ and $I_{p} \simeq 37 \mathrm{~mW}$ the pattern occupies roughly $60 \%$ of the pump area: the CI regime dominates the dynamics of the system [case (ii)].

In conclusion, we have experimentally investigated the dynamics of pattern formation in a tilted photorefractive single-feedback system. The geometry, transverse phase velocity, and dynamics of the forming patterns are strongly influenced by the transverse shift, the mirror distance and the pump beam intensity. When the virtual tilted mirror is located inside the medium, different patterns are observed and an inversion of the pattern transverse phase velocity can be found for particular values of the tilt angle. The signatures of convective and absolute instabilities have been identified for the first time in a photorefractive system, in a similar way to those observed in thin Kerr-type media although the physics that rules the nonlinear light-matter interaction is different. It confirms that the behaviors are general and do not depend on the nature of the involved nonlinearity.

The authors acknowledge the support of the Conseil Régional de Lorraine.
[1] M. Segev and D. N. Christodoulides, Nat. Photonics 3, 195 (2009).

[2] P. Manneville, Dissipative Structure and Weak Turbulence (Academic Press, San Diego, 1990).

[3] F. T. Arecchi, S. Boccaletti, and P. L. Ramazza, Phys. Rep. 318, 1 (1999).

[4] L. A. Lugiato and R. Lefever, Phys. Rev. Lett. 58, 2209 (1987).

[5] G. D’Alessandro and W. J. Firth, Phys. Rev. Lett. 66, 2597 (1991).

[6] K. Staliunas, Opt. Commun. 91, 82 (1992).

[7] T. Honda, Opt. Lett. 18, 598 (1993).

[8] T. Ackemann and W. Lange, Phys. Rev. A 50, R4468 (1994).

[9] A. V. Mamaev and M. Saffman, Opt. Lett. 22, 283 (1997).

[10] T. Carmon, M. Soljacic, and M. Segev, Phys. Rev. Lett. 89, 183902 (2002).

[11] M. Haelterman and G. Vitrant, J. Opt. Soc. Am. B 9, 1563 (1992).

[12] G. Grynberg, Opt. Commun. 109, 483 (1994).

[13] P. L. Ramazza, P. Bigazzi, E. Pampaloni, S. Residori, and F. T. Arecchi, Phys. Rev. E 52, 5524 (1995).

[14] P. L. Ramazza, S. Boccaletti, A. Giaquinta, E. Pampaloni, S. Sonia, and F. T. Arecchi, Phys. Rev. A 54, 3472 (1996).

[15] J. P. Seipenbusch, T. Ackemann, B. Schapers, B. Berge, and W. Lange, Phys. Rev. A 56, R4401 (1997).
[16] R. J. Briggs, Electron-Stream Interaction with Plasmas (MIT Press, Cambridge, MA, 1964).

[17] M. Santagiustina, P. Colet, M. San Miguel, and D. Walgraef, Phys. Rev. Lett. 79, 3633 (1997).

[18] F. Papoff and R. Zambrini, Phys. Rev. Lett. 94, 243903 (2005).

[19] R. Zambrini and F. Papoff, Phys. Rev. Lett. 99, 063907 (2007).

[20] E. Louvergneaux, C. Szwaj, G. Agez, P. Glorieux, and M. Taki, Phys. Rev. Lett. 92, 043901 (2004).

[21] G. Agez, P. Glorieux, M. Taki, and E. Louvergneaux, Phys. Rev. A 74, 043814 (2006).

[22] A. Mussot, E. Louvergneaux, N. Akhmediev, F. Reynaud, L. Delage, and M. Taki, Phys. Rev. Lett. 101, 113904 (2008).

[23] F. Papoff and R. Zambrini, Phys. Rev. A 79, 033811 (2009).

[24] N. Marsal, D. Wolfersberger, M. Sciamanna, G. Montemezzani, and D. Neshev, Opt. Lett. 33, 2509 (2008).

[25] C. Denz, M. Schwab, M. Sedlatschek, T. Tschudi, and T. Honda, J. Opt. Soc. Am. B 15, 2057 (1998).

[26] N. V. Kukhtarev, V. B. Markov, S. G. Odulov, M. S. Soskin, and V. L. Vinetskii, Ferroelectrics 22, 961 (1979).

[27] M. Santagiustina, P. Colet, M. San Miguel, and D. Walgraef, J. Opt. B 1, 191 (1999).

[28] G. Agez, C. Szwaj, E. Louvergneaux, and P. Glorieux, Phys. Rev. A 66, 063805 (2002). 